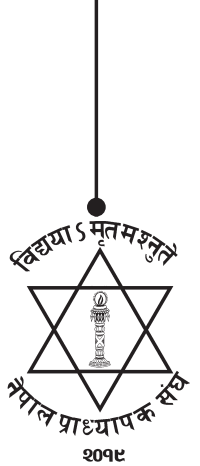

NJ: NUTA

\title{
Development of Computational Research Methods and Application in Chemistry
}

\author{
Deba Bahadur Khadka \\ Professor, Central Department of Chemistry, \\ Tribhuvan University, Kirtipur, Kathmandu \\ Email for correspondence: khadkadeba@yahoo.com
}

\begin{abstract}
The development of computational research methods and its application in chemistry has been described in this paper. Based on theoretical review on computational research, the study applied experimental research method. The discussion of findings showed that, the development of computational research methods have both advantages and disadvantages that have clearly highlighted in this study.
\end{abstract}

Key words: Computational research, Hartree-Fock calculations, density-functional theory, geometry optimization and electronic structure.

\section{Introduction}

Computational Chemistry can be described as chemistry performed using computers rather than chemicals. It covers a broad range of topics including: cheminformatics, Statistical mechanics, molecular mechanics, semi-empirical methods etc. In the history of quantum mechanics, the first theoretical calculations in chemistry were reported by Walter Heitler and Fritz London in 1927. In 1935, the early development of computational quantum chemistry include by Linus Pauling and E. Bright Wilson's. Introduction to Quantum Mechanics with Applications to Chemistry, Eyring, Walter and Kimball's in1944, Quantum Chemistry by Heitler's in 1945,Elementary Wave Mechanics with Applications to Quantum Chemistry and later Coulson's in 1952.

In the 1940s, the development of efficient computer technology the solutions of elaborate wave equations for complex atomic systems began to be a realizable objective. In the early 1950 s, the first semi-empirical atomic orbital calculations have been studied. Theoretical chemists became extensive users of the early digital computers. Smith and Sutcliffe was one such user in the United Kingdom (Smith\& Sutcliffe, 1999).The firstab initio Hartree-Fock calculations on diatomic molecules were carried out in 1956 at MIT using a basis set of Slater orbitals.

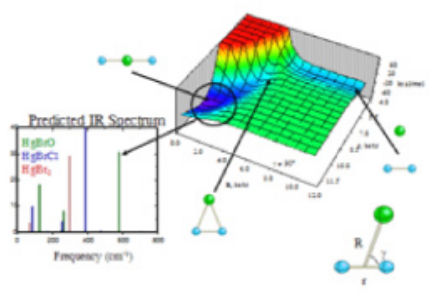

zigure 1: Dynarnies and Spectroscopy of the reactiens of Hz and Halogens. 
In 1960, a systematic study using a minimum basis set and the first calculation with a larger basis set were published by Ransil and Nesbet for diatomic molecules (Schaefer, 1972). The first polyatomic calculations using Gaussian orbitals were carried out in the late 1950s. The first configuration interaction calculations were carried out in Cambridge on the EDSAC computer in the 1950s using Gaussian orbitals by Boys and coworkers (Boys, Cook, Reeves \& Shavitt, 1956).In 1971, when a bibliography of ab initio calculations was published (Richards, Walker \& Hinkley, 1971) the largest molecules included were naphthalene and azulene (Preuss, 1968; Buenker \& Peyerimhoff, 1969). Schaefer have been published the summary of many earlier research developments inab initiotheory (Schaefer, 1984). In 1964, Huckel method calculations using a simple linear combination of atomic orbitals (LCAO) method for the determination of electron energies of molecular orbitals of $\pi$ electrons in conjugated hydrocarbon systems of molecules ranging in complexity from butadiene and benzene to ovalence were generated on computers at Berkeley and Oxford (Streitwieser, Brauman \& Coulson, 1965). In 1960s, these empirical methods were replaced by semi-empirical methods such as CNDO (Pople \& Beveridge, 1970).

In the early 1970s, efficient abinitio computer programs such as ATMOL, Gaussian, IBMOL and POLYAYTOM, began to be used to speed up abinitio calculations of molecular orbitals. Among these four programs, only GAUSSIAN, now massively expanded, is still in use, but many other programs are now in use. At the same time, the methods of molecular mechanics such as MM2 were developed by Norman (Norman, 1977). One of the first mentions of the term "computational chemistry" was found in the 1970 book Computers and Their Role in the Physical Sciences by Sidney Fernbach and Abraham Haskell Taub, where they state "It seems, therefore, that 'computational chemistry' can finally be more and more of a reality (Haskell, 1970). During the 1970s, widely different methods began to be seen as part of a new emerging discipline of computational chemistry (RSAS, 1998). Computational chemistry has featured in a number of Nobel Prize awards, most notably in 1998 and 2013. The Journal of Computational Chemistry was first published in 1980. Walter Kohn, "for his development of the density-functional theory", and John Pople, "for his development of computational methods in quantum chemistry", received the 1998 Noblel Prize in Chemistry (RSAS, 2013). Martin Karplus, Michael Levitt and Alrieh Warshel received the 2013 Nobel Prize in Chemistry for "the development of multiscale models for complex chemical systems (JMGM). The objectives of this paper are to report the brief review development of computational research methods and application in chemistry.

\section{Advantages}

- Calculations are easy to perform, whereas experiments are often difficult.

- Calculations are becoming less costly, whereas experiments are becoming more expensive.

- Calculations can be performed on any system, even those that don't exist, whereas many experiments are limited to relatively stable molecules.

- Calculations are safe, whereas many experiments have an intrinsic danger associated with them.

- Introduction Hartree Fock Theory Configuration Interaction Background.

\section{Disadvantages}

- Calculations are too easy to perform; many black-box programs are available to the uninitiated.

- Calculations can be very expensive in terms of the amount of time required.

- Calculations can be performed on any system, even those that don't exist.

NUTA JOURNAL, 5 (1\&2), 2074 : ISSN: 2616 - 017x 
- Computational chemistry is not a replacement for experimental studies, but plays an important role in enabling chemists to explain and rationalize known chemistry.

- Explore new or unknown chemistry.

\section{Applications}

The computational chemistry is usually used when a mathematical method is sufficiently well developed that it can be automated for implementation on a computer. In theoretical chemistry, chemists, mathematicians and physicists develop algorithms and computer programs to predict atomic and molecular properties and reaction paths for chemicals reactions. Computational chemists, in contrast, may simply apply existing computer programs and methodologies to specific chemical titles. There are two different aspects to computational chemistry. Computational studies can be carried out to find a starting point for a laboratory synthesis, or to assist in understanding experimental data such as the position and source of spectroscopic peaks. Computational studies can be used to predict the

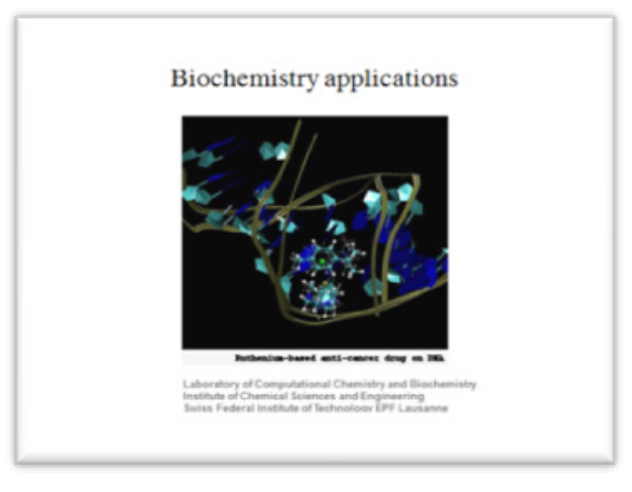
possibility of so far entirely unknown molecules or to explore reaction mechanisms that are not readily studied by experimental means. Thus, computational chemistry can assist the experimental chemist or it can challenge the experimental chemist to find entirely new chemical objects. Several major areas may be distinguished within computational chemistry: The prediction of the molecular structure of molecules by the use of the simulation of forces or more accurate quantum chemical methods to find stationary points on the energy surface as the position of the nuclei is varied. Storing and searching for data on chemical entities. Computational approaches to help in the efficient synthesis of compounds.

Computational approaches to design molecules that interact in specific ways with other molecules e.g. catalysis and drug design. Almost every aspect of chemistry can be described in a qualitative or approximate quantitative computational scheme. Molecules consist of nuclei and electrons, so the methods of quantum mechanics apply. Computational chemists often attempt to solve the nonrelativistic Schrodinger equation with relativistic corrections added, although some progress has been made in solving the fully relativistic Dirac equation. In principle, it is possible to solve the Schrodinger equation in either its time-dependent or time-independent form as appropriate for the problem in hand; in practice, this is not possible except for very small systems. Thus, a great number of approximate methods strive to achieve the best trade-off between accuracy and computational cost. Accuracy can always be improved with greater computational cost. Significant errors can present themselves in $a b$ initio models comprising many electrons, due to the computational expense of full relativisticinclusive methods. This complicates the study of molecules interacting with high atomic mass unit atoms such as transitional metals and their catalytic properties. Present algorithms in computational chemistry can routinely calculate the properties of molecules that contain up to about 40 electrons with sufficient accuracy. Errors for energies can be less than a few $\mathrm{kJ} / \mathrm{mol}$. For geometries, bond lengths can be predicted within a few picometres and bond angles within 0.5 degrees. The treatment of larger 
molecules that contain a few dozen electrons is computationally tractable by approximate methods such as density functional theory (DFT).

A single molecular formula can represent a number of molecular isomers. Each isomer is a local minimum on the energy surface is called potential energy surface created from the total energy i.e., the electronic energy, plus the repulsion energy between the nuclei as a function of the coordinates of all the nuclei. A stationary point is geometry such that the derivative of the energy with respect to all displacements of the nuclei is zero. A local energy minimum is a stationary point where all such displacements lead to an increase in energy. The local minimum that is lowest is called the global minimum and corresponds to the most stable isomer. If there is one particular coordinate change that leads to a decrease in the total energy in both directions, the stationary point is transition structure and the coordinate is the reaction coordinate. This process of determining stationary points is called geometry optimization. The determination of molecular structure by geometry optimization became routine only after efficient methods for calculating the first derivatives of the energy with respect to all atomic coordinates became available. Evaluation of the related second derivatives allows the prediction of vibrational frequencies if harmonic motion is estimated. It also allows for the characterization of stationary points.

The frequencies are related to the eigenvalues of the Hessian matrix, which contains second derivatives. If the eigenvalues are all positive, then the frequencies are all real and the stationary point is a local minimum. If one eigenvalue is negative i.e., an imaginary frequency, then the stationary point is a transition structure. If more than one eigenvalue is negative, then the stationary point is a more complex one, and is usually of little interest. When one of these is found, it is necessary to move the search away from it if the experimenter is looking solely for local minima and transition structures. The total energy is determined by approximate solutions of the timedependent Schrodinger equation, usually with no relativistic terms included, and by making use of the Born-Oppenheimer

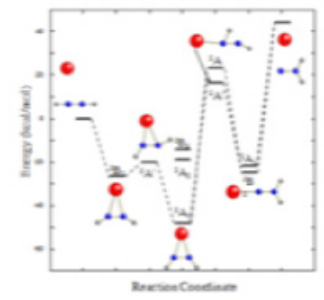
approximation, which allows for the separation of electronic and nuclear motions, thereby simplifying the Schrödinger equation. This leads to the evaluation of the total energy as a sum of the electronic energy at fixed nuclei positions and the repulsion energy of the nuclei. The first-principles methods are being widely used to study the electronic structure of solids, surfaces, or clusters. With the recent developments in the computer speed and capacity, the physical and chemical properties of the complex systems like macromolecules of biological importance such as DNA and RNA have been studied using the first-principles methods (Bhattrai, 2007). The first-principles can be classified into three main categories: the Hartree-Fock approach, the density functional approach and the quantum Monte-Carlo approach (Bhattrai, 2007; Neupane, 2009). In what ways, we briefly discuss about the Hartree-Fock self-consistent method, Møller-Plesset perturbation method, configuration interaction and density functional theory.

According to Hartree's SCF method of the atom, the motion of the each electron in effective field of the N-1 others is governed by a one-particle Schrödinger equation. The self-consistency of 
the electronic charge distribution with its own electrostatic field leads to a set of coupled integrodifferential equations known as the Hartree equations for the $\mathrm{N}$ one particle wave functions (Blinder, 1965).This method fails to satisfy the antisymmetry principle (Sherril, 2000). This method was modifyed by the Slater and Fock independently. The HF theory starts from a single antisymmetry spin-orbital product, an approximation which has far dominated molecular theory. Usually, the possible MO's to use in such wave functions are determined variationally by the SCF method. It is, however, unnecessary to determine the orbitals themselves since in this approximation the wave function is completely determined by the 1-electron density matrix and this may be calculated directly by a suitable iterative method (McWeeny,

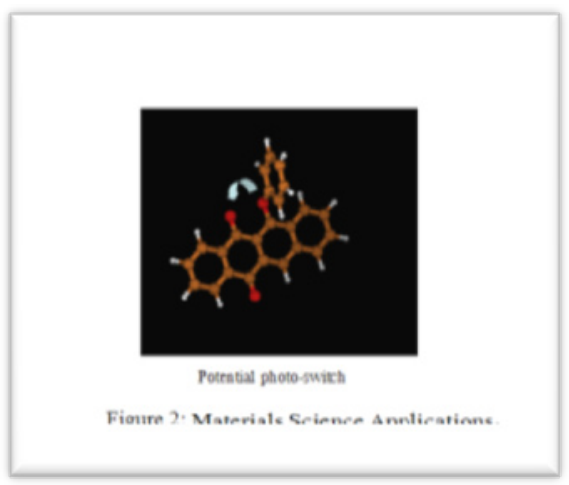
1960). The HF method provides approximate solutions to the Schrodinger equation by replacing the real electron-electron interaction with an average interaction. The Electron Correlation (EC) is energy difference between the HF and the lowest possible energy in each basis set. It is due to a correlation between the motions of electrons. For systems and states where correlation effects are important, the HF results will not be satisfactory. Quantum Mechanics methods have been developed to include some effects of Electron Correlation (Van, 2006).

The main correlation correction methods are Møller-Plesset perturbation theory and configuration interaction. In MP theory, HF energy is improved by obtaining a perturbation expansion from HF wavefunction for the correlation energy. However, MP calculations are not variational and can produce energy value below true energy due to overcorrection the minimal correlation is the second order MP2 method (Neupane, 2009). Third order MP3 and fourth order MP4 calculations are also possible. The results of MP4 calculation is equivalent to a CISD calculation. MP5 and higher calculations are seldom carried out due to the high computation time (Van, 2006). Another method employs linear combinations of the Slater determinants involving excited electronic states of the molecule instead of using just a single Slater determinant involving the lowest orbitals for the molecular wave function. This approach is called configuration interaction, and it is the most complete treatment of electronic structure possible for a given basis set (McQuarrie \& Simon, n.d.). The full CI calculations with large basis sets are usually carried out for only the smallest molecules (Cramer, 2007).

Another first-principles approach to study the electronic structure and property of the many electron systems is the DFT. The Thomas-Fermi equations, together with an assumed variational principle, represented the first effort to define a density functional theory (Cramer, 2007). To describe interacting electrons, the extremely simple three-dimensional electron density can be used as the basic variable within DFT (Cohen, Mori-Sanchez \& Yang, 2008). The exchange correlation energy is expressed as a functional of the resulting electron density, and the electronic states are solved selfconsistently as in HF approximation (Neupane, 2009). The success of the DFT is based on the quality of approximation for the energy functional. The construction of the accurate approximations depends on the theoretical formulation (Yang, Zhang \& Ayers, 2000). Some of the results of application reported in the literature by different group are shown below (Ben, 2006). It has been known that calculations 
can be performed on any system even those that don't exist, whereas many experiments are limited to relatively stable molecules. The discussion and comparison show that theoretical and experimental research works have both advantages and disadvantages.

\section{References}

Ben, S. (2006). Introduction to Computational Quantum Chemistry. Chemical, 334. Spring.

Bhattrai, G. (2007). Equilibrium Configurations of $\mathrm{H}_{3} \mathrm{~N}-\mathrm{BF}_{3}, \mathrm{H}_{3} \mathrm{~N}-\mathrm{BCl} \mathrm{l}_{3}$, and $\mathrm{H}_{3} \mathrm{~N}-\mathrm{BF}{ }_{3}$, [Unpublished MA Thesis]. Submited to the Central Department of Physics, Tribhuvan University, Kirtipur, Nepal.

Blinder, S. M. (1965). American Journal of Physics, 33 (6), 431.

Boys, S. F., Cook, G. B., Reeves, C. M. \& Shavitt, I. (1956). “Automatic Fundamental Calculations of Molecular Structure. Nature, 178 (2), 1207.

Buenker, R. J. \& Peyerimhoff, S. D. (1969). Ab initio SCF calculations for azulene and naphthalene. Chemical Physics Letters, 3, 37.

Cohen, A. J., Mori-Sanchez, P. \& Yang, W. (2008). Science 321, 792. Retrieved from www.sciencemag. org

Cramer, C. J. (2007). Essentials of Computational Chemistry Theories and Model, (2nd ed.). West Sussex PO198SQ, England: John Wiley and Sons, Limited.

Haskell, A. (1970). Computers and Their Role in the Physical Sciences. Fernbach, Sidney and Taub: Routledge.

JMGM. Retrieved from http://www.bio-balance.com/JMGM_article.pdf

McQuarrie, D. A. \& Simon, J. D. (n. d.). Physical Chemistry A Molecular Approach”, First South Asian Edition, University Science Books, 55D Gate Five Road, Sausalito, CA 94965

McWeeny, R. (1960). Rev. Mod. Physics, 32 (2), 335.

Neupane, T. R. (2009). Study of Electron Correlation Effects on the Stability of Ozone Molecule, [Unpublished MA Thesis]. Submitted to the Central Department of Physics, Tribhuvan University, Kirtipur, Nepal.

Norman, A. (1977). Conformational Analysis: A Hydrocarbon Force Field Utilizing V1 and V2 Torsional Terms. Journal of the American Chemical Society, 99 (25), 8127.

Pople, J. A. \& Beveridge, D. L. (1970). Approximate molecular orbital theory. New York: McGraw Hill.

Preuss, H. (1968). International Journal of Quantum Chemistry, 2, 651.

Richards, W. G., Walker, T. E. \& Hinkley, R. K. (1971). A bibliography of ab initiomolecular wave functions. Oxford: Clarendon Press.

Royal Swedish Academy of Sciences (RSAS). (1998). Reviews in Computational Chemistry. Vol. 1, Preface.

Royal Swedish Academy of Sciences (RSAS). (2013). The Nobel Prize in Chemistry. Retrieved October 9, 2013.

Schaefer, H. F. (1972). The electronic structure of atoms and molecules. Reading, Massachusetts: Addison-Wesley Publishing Company.

Schaefer, H. F. (1984). Quantum Chemistry. Oxford: Clarendon Press. 
Sherril, C. D. (2000). An Introduction to Hartree-Fock Molecular Orbital Theory, School of Chemistry and Biochemistry, Georgia Institute of Technology.

Smith, S. J. \& Sutcliffe, B. T. (1997). The development of Computational Chemistry in the United Kingdom. Reviews in Computational Chemistry, 10, 271.

Streitwieser, A., Brauman, J. I. \& Coulson, C. A. (1965). Supplementary tables of molecular orbital calculations. Oxford: Pergamon Press.

Van, T. P. (2006). Ab Initio Calculations of Intermolecular Potentials, Prediction of Second Virial Coefficients for Dimers $\mathrm{H}_{2}-\mathrm{H}_{2}, \mathrm{H}_{2}-\mathrm{O}_{2}, \mathrm{~F}_{2}-\mathrm{F}_{2}$ and $\mathrm{H}_{2}-\mathrm{F}_{2}$, and Monte Carlo simulations of the Vapour-Liquid Equilibria for Hydrogen and Fluorine, [Unpublished PhD Dissertation]. Submitted to the Institute of Physical Chemistry, University of Colgone, Köln, Germany.

Yang, W., Zhang, Y. \& Ayers, P. W. (2000). Physics Review. Letters, 84 (22), 5172. 